\title{
FATORES AMBIENTAIS QUE INFLUENCIAM O PESO À DESMAMA, ANO E SOBREANO EM BOVINOS DA RAÇA NELORE MOCHA, NO SUDOESTE DE MATO GROSSO DO SUL - BRASIL
}

\author{
(Environmental factors that influence the weaning weight, year weight and weight at 18- \\ months in Nelore Mocho cattle in the southwest of Mato Grosso do Sul - Brazil)
}

\author{
CONCEIÇÃO, F.M.'; FERRAZ FILHO, P.B. ${ }^{2}$ SILVA, L.O.C. ${ }^{3}$; BRAGANÇA, V.L.C. ${ }^{4}$; SOUZA, J.C. ${ }^{5}$
}

1'Aluna do Mestrado em Ciência Animal pela UFMS, fernandamartinsc@terra.com.br;

2Professo Adjunto DCN/UFMS,pbferraz@ceul.ufms.br;

3Pesquisador EMBRAPA Gado de Corte. locs@cnpgc.embrapa.br;

${ }^{4}$ Professor Adjunto DMV/UCDB, victorcordoba@terra.com.br;

${ }^{5}$ Professor Adjunto DZ/UFPR, jcs@ufpr.br.

RESUMO - Animais da raça Nelore Mocha, criados em regime de pastagens, na região sudoeste do estado de Mato Grosso do Sul, no período de 27 anos, permitiu a confecção de um banco de dados com 21.919 observações de pesos a desmama (P205), ao ano (P365) e ao sobreano (P550). Estas foram analisadas pelo método dos quadrados mínimos, para verificar a influência dos fatores ambientais sobre as mesmas. O modelo estatístico incluiu os efeitos fixos de ano e mês de nascimento do bezerro, sexo, região e a covariável de idade da vaca ao parto (linear e quadrático). A média observada do peso aos 205 dias de 9.256 observações foi de 181,30 $\pm 23,29 \mathrm{~kg}$, para peso aos 365 dias, de 7.786 animais, a média foi $244,51 \pm 35,49 \mathrm{~kg}$ e para o peso aos 550 dias, 4.877 observações a média do peso foi de $327,54 \pm 47,80 \mathrm{~kg}$. Os efeitos foram significativos $(\mathrm{P}<0,001)$, as diferenças entre os anos e meses de nascimento e regiões foram evidentes para as três características. A idade da vaca ao parto (linear e quadrático), influenciou apenas os pesos aos 205 e 365 dias, com pesos máximos atingidos as idades de 8,8 e 8,5 anos, respectivamente. A diferença entre os sexos aumentou gradativamente com a idade, sendo a superioridade dos machos em relação às fêmeas de 9,36\% (P205), 13,09\% (P365) e 18,56\% (P550).

Palavras-chave: bovino corte, Bos indicus.

ABSTRACT - Animals of the race Nelore hornless (mocha = sem cornos) in regime of pastures in the three territorial units of the Southwest region of Mato Grosso do Sul, Brazil, in the period of 27 years, were analyzed with the objective of evaluating the environmental and genetic effects on the weight of these animals. Relative data to 21,919 observations of weights at the weaning (W205), at the year (W365) and at 18-month (W550) have been considered. Analyses were accomplished by the method of the minimum squares, to verify the influence of some environmental factors. The statistical model included the fixed effects of year and month from birth, sex, area and the covariable of age of the cow to the childbirth (lineal and quadratic). The observed average of the weight to the 205 days of 9,256 observations was of $181,30 \pm 23,29 \mathrm{~kg}$, for weight to the 365 days with average of 7,786 observations was of $244,51 \pm 35,49 \mathrm{~kg}$ and for the weight to the 550 days of 4,877 observations was of $327,54 \pm 47,80 \mathrm{~kg}$. The effects of them were half significant $(P<0,001)$, the differences between the years and months from birth and areas were evident for the three characteristics. The age of the cow at the childbirth (lineal and quadratic), only influenced the weights to the 205 and 365 days, with maximum weights reaching at the ages of 8,8 and 8,54 years, respectively. The difference between sexes increased gradually with the age, being the superiority of the males in relation to the females of 9,36\% (W205), 13,09\% (W365) and 18,56\% (W550).

Key-words: Beef cattle, Bos indicus. 


\section{Introdução}

A produção de bovinos de corte no Brasil avança a cada ano em função do aumento crescente da população mundial. Segundo o IBGE (2003), o Brasil possui mais de 195 milhões de cabeças, e na busca por maximizar o sistema de produção e se ajustar às mudanças que acontecem atualmente no planeta, vem utilizando-se de novas tecnologias em seus programas de manejo e melhoramento animal (FERRAZ FILHO, 2001). No Brasil devido à diversidade de condições ecológicas e os mais diferentes sistemas de produção, existe à necessidade de estudos sobre os índices produtivos dos rebanhos por regiões, contribuindo para o melhoramento de sua pecuária.

Além dos fatores ambientais referentes ao local de criação (clima, solo, pluviosidade, etc), fatores ambientais, como rebanho, ano e época de nascimento, sexo e idade da vaca ao parto, também são relatados como importantes fontes de variação das características de produção em gado de corte (FERRAZ FILHO et al., 2001). Um melhor conhecimento das causas que influenciam características produtivas, como os pesos corpóreos, e em especial dos parâmetros genéticos e de ambiente, são de relevante importância para um melhor conhecimento dos fatores que interferem no crescimento/ desenvolvimento dos rebanhos bovinos de corte no Brasil (GRESSLER, 1998).

O objetivo deste trabalho foi avaliar a influencia dos efeitos do meio sobre o peso dos animais da raça Nelore Mocha, aos 205 (peso a desmama), 365 (peso ao ano) e 550 (peso ao sobreano) dias de idade, criados a pasto, na região sudoeste do estado de Mato Grosso do Sul.

\section{Materiais e Métodos}

Os dados utilizados para o presente estudo referem-se às informações de pesos de bovinos da raça Nelore Mocha, obtida pelos técnicos da Associação Brasileira de Criadores de Zebu (ABCZ). Estas informações integram o Sistema de Apoio ao Melhoramento Genético do Zebu (Sis-Zebu), mantido pelo convênio ABCZ/EMBRAPA, as quais foram gentilmente cedidas pelo Centro Nacional de Pesquisa de Gado de Corte da EMBRAPA, localizada em Campo Grande/ MS.

Analisou-se dados de 21.919 registros de animais pertencentes a mesorregião geográfica do Sudoeste do Estado do Mato Grosso do Sul, definidas pelo IBGE com três unidades territoriais, Bodoquena (Região I), Dourados (Região II) e lguatemi (Região III). Os animais nasceram no período de 1975 a 2001. Avaliaram-se 9.256 progênies de 340 touros para peso aos 205 dias; 6.923 progênies de 321 touros para peso aos 365 dias e 4.498 progênies de 261 touros para o peso aos 550 dias.

A Região I foi composta por 1.159 registros, sendo, 433 animais para $\mathrm{P} 205,485$ para $\mathrm{P} 365$ e 241 para P550. A Região II continha 9.520 registros, sendo, 4.028 para P205, 3.397 para P365 e 2.095 para P550. A Região II foi composta 11.240 registros, sendo, 4.795 para P205, 3.904 para P365 e 2.541 para P550.

Os pesos foram ajustados por interpolação para as idades padrões, utilizando-se as pesagens mensais mais próximas daquelas idades, FERRAZ FILHO (2001).

As análises de variância foram conduzidas pelo Método dos Quadrados Mínimos, procedimento GLM do programa SAS, (2001). O modelo estatístico continha os efeitos fixos de ano e mês de nascimento, sexo, região e a covariável de idade da vaca ao parto (linear e quadrático), além do efeito aleatório de touro e do erro, conforme descrito a seguir:

$$
Y_{i j k l m n}=\mu+T_{i}+A_{j}+M_{k}+S_{l}+R_{m}+b_{1}\left(X_{i j k l m n}-\bar{X}\right)+b_{2}\left(X_{j k l m n}-\bar{X}\right)^{2}+e_{i j k l m n}
$$

em que:

$Y_{i j k l m n}=$ variável dependente (P205) do nésimo filho do touro $\mathrm{i}$, dentro do ano j, nascido no mês $\mathrm{k}$ e de sexo $\mathrm{l} ; \mu$ = média geral para as características analisadas; $T_{i}=$ efeito aleatório do reprodutor i, $A_{j}=$ efeito fixo do ano de nascimento j; $M_{k}=$ efeito fixo do mês $\mathrm{k}$ de nascimento; $S_{l}=$ efeito fixo do sexo da cria I; $R_{m}=$ efeito da região $\mathrm{m} ; X_{i j k l m n}=$ idade da vaca ao parto; $b_{1}=$ coeficiente de regressão para idade linear; $b_{l}=$ coeficiente de regressão para idade quadrática; e $e_{j k l m n}=$ erro aleatório, normal e independentemente distribuído com média zero e variância $=1$. 
Fatores ambientais que influenciam o peso à desmama, ano e sobreano em bovinos da raça nelore mocha...

\section{Resultados e Discussão}

A média observada para o peso aos 205 dias de idade foi de $181,30 \pm 23,29 \mathrm{~kg}$, com um coeficiente de variação de $12,84 \%$. Para o peso aos 365 dias foi $244,51 \pm 35,49 \mathrm{~kg}$ com coeficiente de variação de $14,52 \%$, e para o peso aos 550 dias de idade foi 327,54 $\pm 47,80 \mathrm{~kg}$ com coeficiente de variação de e
$14,59 \%$.

O resumo das análises de variância por quadrados mínimos referentes aos pesos estudados está apresentado na TABELA 1, onde se verifica que quase todos os efeitos incluídos no modelo foram significativos $(P<0,001)$, com exceção da covariável idade da vaca ao parto (linear e quadrática), para o peso aos 550 dias de idade.

TABELA 1 - RESUMO DAS ANÁLISES DE VARIÂNCIA, POR QUADRADOS MÍNIMOS, DOS PESOS AOS 205 (P205) DIAS DE IDADE DE BOVINOS NELORE MOCHO NA REGIÃO SUDOESTE DE MATO GROSSO DO SUL.

\begin{tabular}{|c|c|c|c|c|c|c|}
\hline \multirow{2}{*}{ Fonte de Variação } & \multicolumn{2}{|c|}{ P205 } & \multicolumn{2}{|c|}{ P365 } & \multicolumn{2}{|c|}{ P550 } \\
\hline & $\mathrm{GL}$ & QM & $\mathrm{GL}$ & QM & $\mathrm{GL}$ & QM \\
\hline Touro & 339 & $6759,82^{*}$ & 320 & $9.292,64^{*}$ & 260 & $12.000,81^{*}$ \\
\hline Ano & 26 & $7067,75^{\star}$ & 26 & $11.744,06^{*}$ & 25 & $15.035,65^{\star}$ \\
\hline Nascimento & 11 & $45190,63^{*}$ & 11 & $25.280,82^{*}$ & 11 & $31.344,08^{*}$ \\
\hline Mês & 1 & $537111,33^{*}$ & 1 & $1.652 .305,85^{\star}$ & 1 & $3.155 .657,76^{*}$ \\
\hline $\begin{array}{l}\text { Nascimento } \\
\text { Sexo }\end{array}$ & 2 & $28589,99^{*}$ & 2 & $66.734,58^{*}$ & 2 & $23.534,98$ * \\
\hline Região de Criação & 1 & $8828,57^{*}$ & 1 & $80.747,17^{*}$ & 1 & $16.656,69 \mathrm{NS}$ \\
\hline $\begin{array}{l}\text { Idade da Vaca } \\
\text { Linear }\end{array}$ & 1 & $67287,17^{*}$ & 1 & $78.616,10^{*}$ & 1 & $18.940,21 \mathrm{NS}$ \\
\hline Quadrática & 8.874 & 542,33 & 6.560 & $1.259,81$ & 4.575 & $2.284,87$ \\
\hline
\end{tabular}

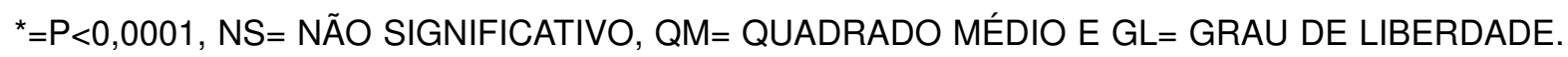

O ano de nascimento influenciou significativamente $(P<0,001)$ todas as características estudadas, TABELA 1. Os animais mais pesados aos 205 dias nasceram no ano de 1976, com 186,99 kg, e os mais leves, no ano de 1975, com 163,23 $\mathrm{kg}$, com média geral de 178,98 kg. O peso aos 365 dias variou de 236,01 $\mathrm{kg}$ em 1998 os mais leves e $270,32 \mathrm{~kg}$ em 1985 os mais pesados, com média geral igual a $251,05 \mathrm{~kg}$. Aos 550 dias o peso variou de $288,65 \mathrm{~kg}$ os mais leves no ano de 2000 , e os mais pesados com 420,08 kg em 1975, com média geral de $323,90 \mathrm{~kg}$.

FIGURA 1 - EFEITO DO ANO DE NASCIMENTO SOBRE O PESO AOS 205 (P205) DIAS DE IDADE EM BOVINOS DA RAÇA NELORE MOCHO, SUDOESTE DE MATO GROSSO DO SUL (19752001).

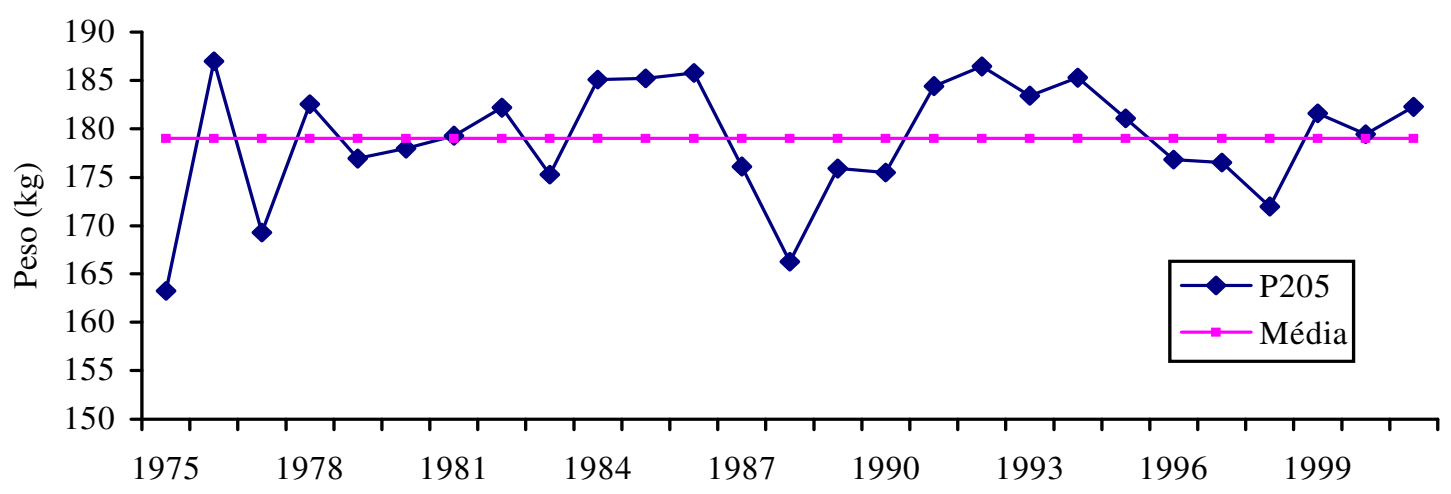


FIGURA 2 - EFEITO DO ANO DE NASCIMENTO SOBRE O PESO AOS 365 (P365) DIAS DE IDADE EM BOVINOS DA RAÇA NELORE MOCHO, SUDOESTE DE MATO GROSSO DO SUL (19752001).

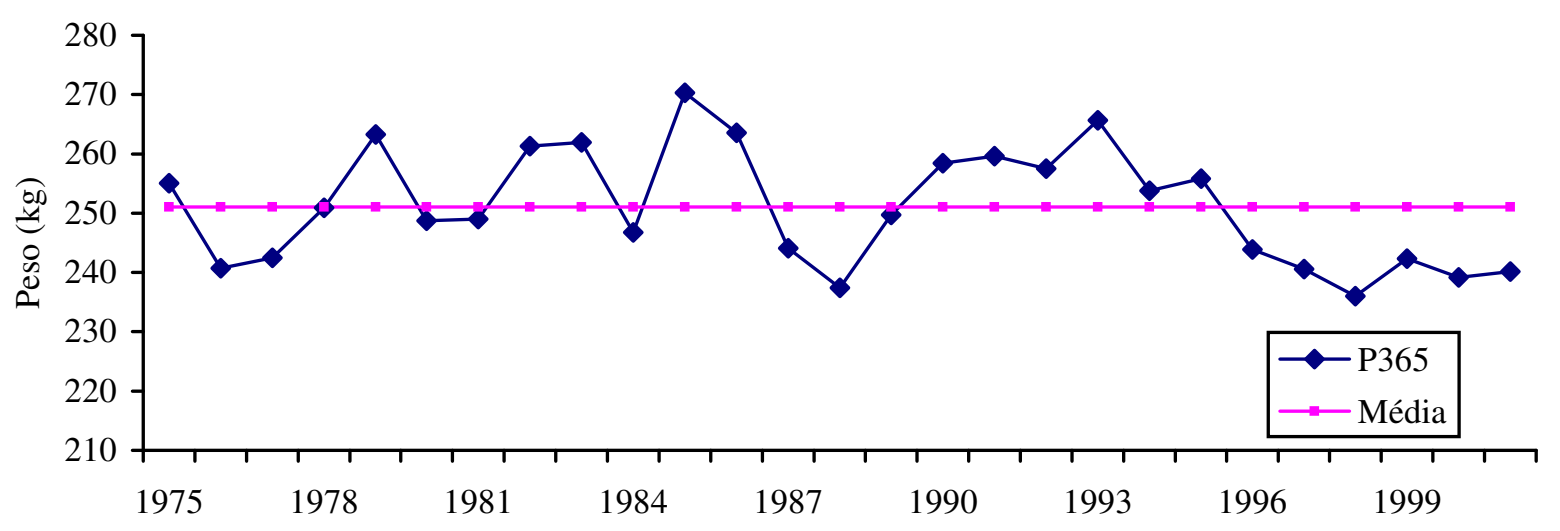

FIGURA 3 -EFEITO DO ANO DE NASCIMENTO SOBRE O PESO AOS 550 (P550) DIAS DE IDADE EM BOVINOS DA RAÇA NELORE MOCHO, SUDOESTE DE MATO GROSSO DO SUL (1975-2000).

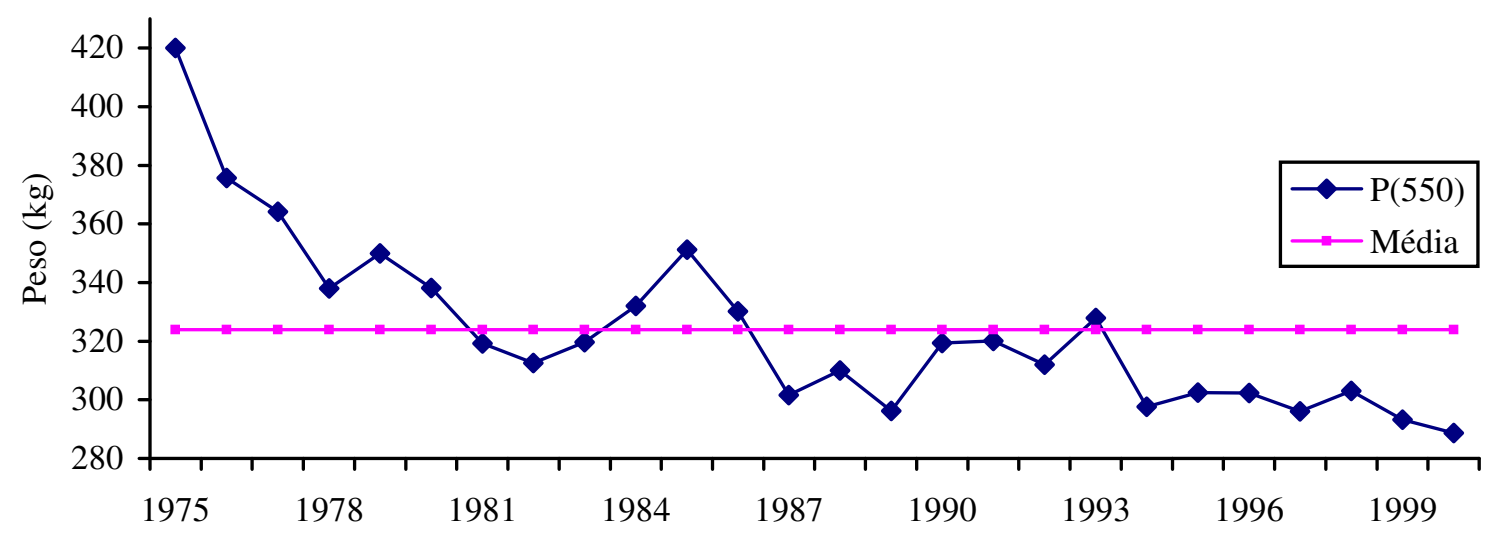

O efeito do mês de nascimento sobre os pesos reflete a variação das condições temporárias do meio, de um mês para outro. A influência do mês de nascimento sobre o peso dos bovinos foi observada por alguns autores, entre eles ELER et al., (1989); MILAGRES et al., (1993), SOUZA et al., (1994), SOUZA et al., (2000) e MALHADO et al. (2001). O mês de nascimento afetou $(P<0,001)$, os pesos aos 205, 365 e 550 dias de idade como observado na TABELA 1. Pode-se observar também uma variação de $15,14 \%$ entre os maiores e os menores pesos aos 205 dias de idade. Os maiores pesos aos 205 dias foram obtidos pelos animais nascidos no período de maio a novembro, com máximo para os meses de junho e setembro. Por outro lado, aqueles que nasceram no período de dezembro a abril foram os que se apresentaram mais leves á idade considerada, com mínimo para os nascidos em março, conforme observado na FIGURA 4.

Para o peso aos 365 dias de idade, encontrou-se uma variação de $8,73 \%$ entre os pesos. Observou-se que o intervalo de nascimento de fevereiro a julho foi o mais favorável para o peso aos 365 dias, com destaque para o mês de junho, enquanto que os meses de agosto a janeiro foram os mais desfavoráveis, sendo novembro o mês com menor peso conforme FIGURA 5, isto provavelmente devido ao fato de os animais nascidos em fevereiro e julho terem passado a fase aguda da seca, dispondo de menor produção de leite materna e durante o período 
Fatores ambientais que influenciam o peso à desmama, ano e sobreano em bovinos da raça nelore mocha...

após a desmama desfrutarem de pastagens fartas e de alto valor nutritivo.

$\mathrm{E}$, para os pesos aos 550 dias de idade (FIGURA 6), observou-se uma variação de $10,35 \%$ entre os valores, sendo o período de junho a dezembro os meses mais favoráveis tendo pico no mês de junho, já os compreendidos entre janeiro e maio foram os meses menos favoráveis, sendo o março o mais desfavorável.

FIGURA 4 - EFEITO DO MÊS DE NASCIMENTO SOBRE O PESO AOS 205 (P205) DIAS DE IDADE, EM BOVINOS DA RAÇA NELORE MOCHO, SUDOESTE DE MATO GROSSO DO SUL (1975-2001).

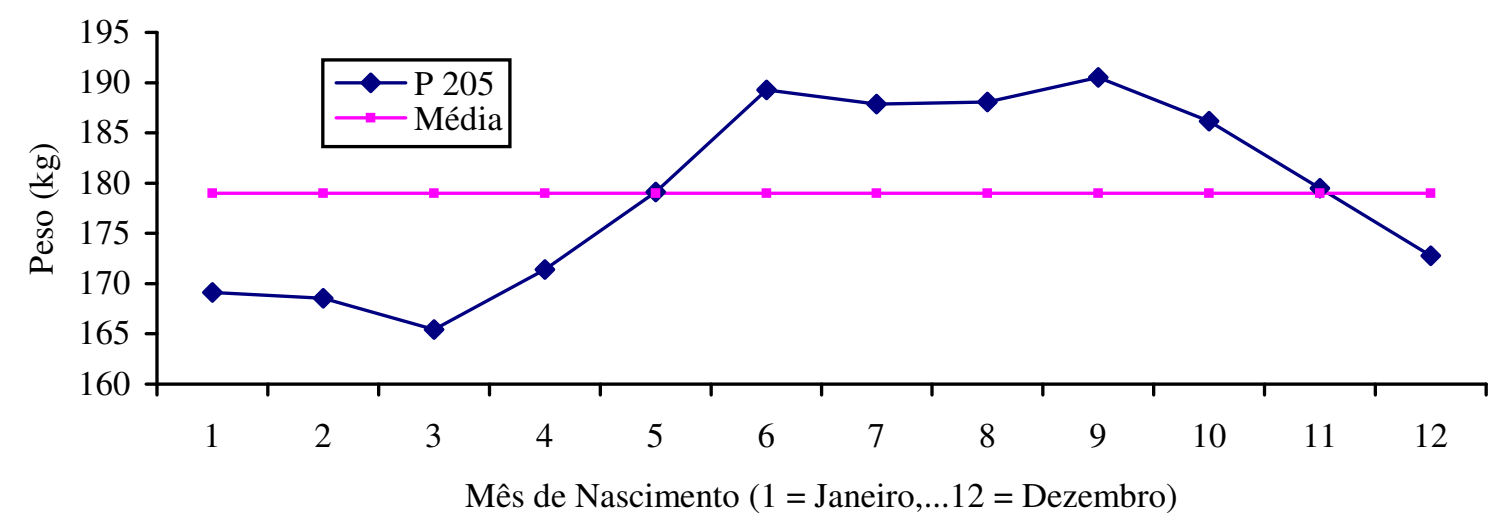

FIGURA 5 - EFEITO DO MÊS DE NASCIMENTO SOBRE O PESO AOS 365 (P365) DIAS DE IDADE, EM BOVINOS DA RAÇA NELORE MOCHA, SUDOESTE DE MATO GROSSO DO SUL (1975-2001).

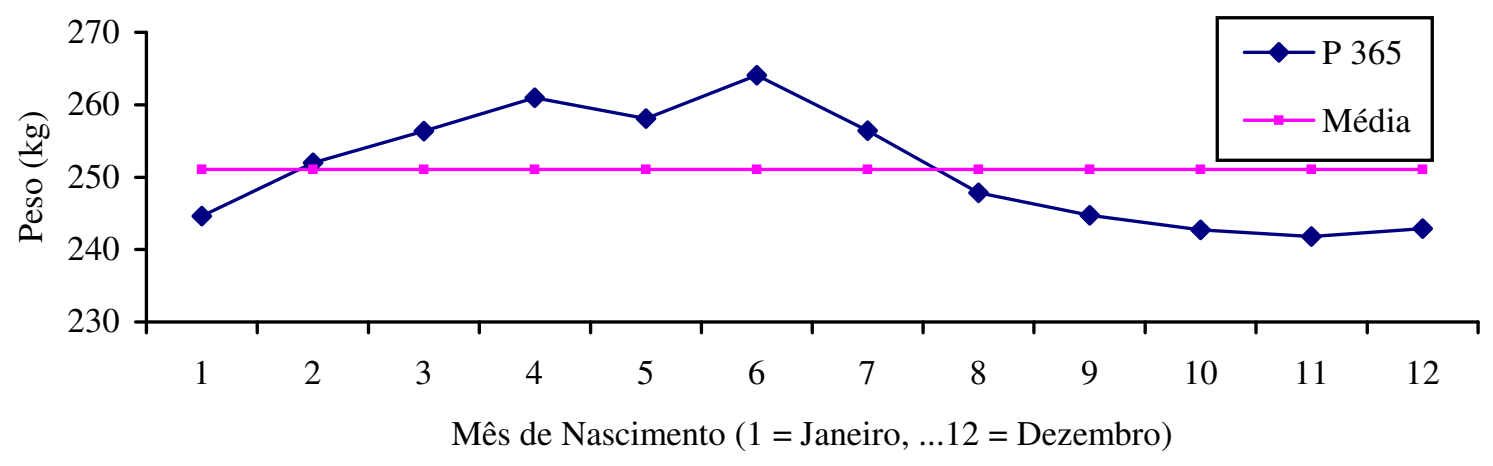

FIGURA 6 - EFEITO DO MÊS DE NASCIMENTO SOBRE O PESO AOS 550 (P550) DIAS DE IDADE, EM BOVINOS DA RAÇA NELORE MOCHA, SUDOESTE DE MATO GROSSO DO SUL (1975-2000).

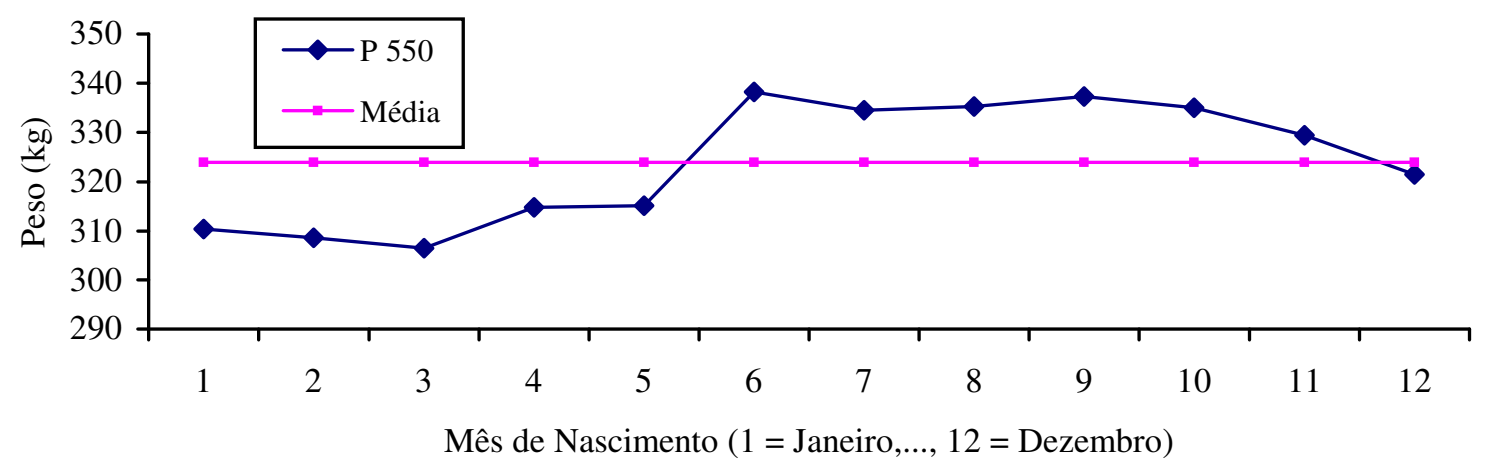


O sexo do bezerro mostrou efeito significativo $(\mathrm{P}<0,001)$ sobre todos os pesos estudados, conforme TABELA 1. Os machos apresentaram superioridade em relação às fêmeas, o que pode ser atribuído à sua capacidade genética em apresentar maiores índices de crescimento pré e pós-natal, possivelmente devido a fatores hormonais (MARTINS et al., 1996). A diferença entre os sexos, como se observa na FIGURA 7, aumentou gradativamente com a idade, sendo a superioridade dos machos em relação às fêmeas de 9,36\% (P205), 13,09\% (P365) e $18,56 \%$ (P550), concordando com os resultados obtidos por EUCLIDES FILHO et al., (1991), SOUZA et al., (1994), FERRAZ FILHO, (2001) e SOUZA et al., (2000).

FIGURA 7 - EFEITO DO SEXO SOBRE O PESO AOS 205 (P205), 365 (P365) E AOS 550 (P550) DIAS DE IDADE EM BOVINOS DA RAÇA NELORE MOCHO, SUDOESTE DE MATO GROSSO DO SUL (1975-2001).

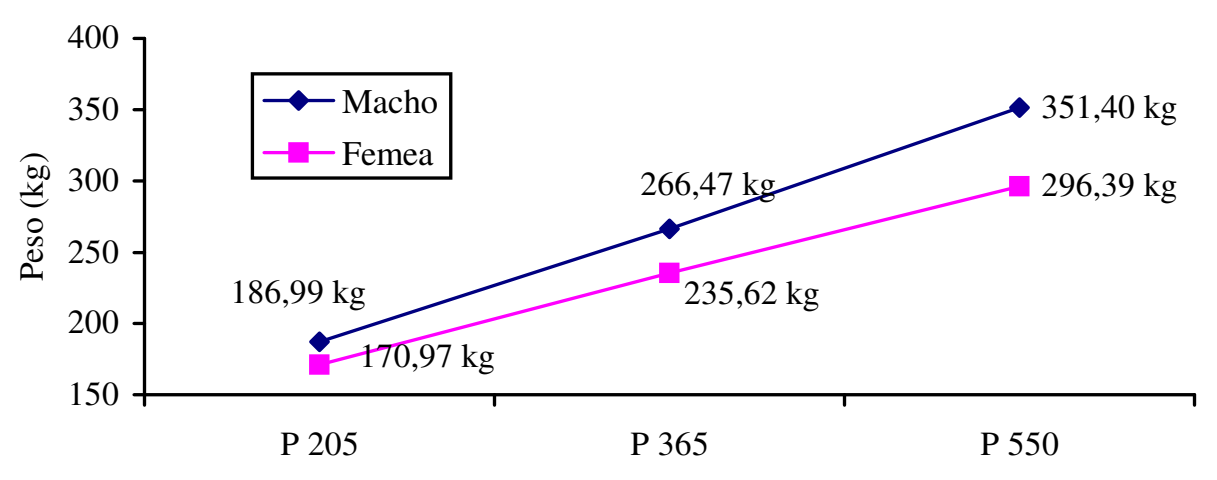

Efeito do Sexo

Os resultados obtidos, considerando o efeito do sexo, sobre as características estudadas, assemelham-se aos reportados por SILVA (1990), SOUZA et al., (1994), SOUZA e RAMOS (1995), MASCIOLI et al., (1997) e SOUZA et al., (2000). Os dimorfismos sexuais crescentes também foram observados por NOBRE et al., (1985), ROSA et al., (1986), SILVA et al., (1987), SILVA (1990), FERRAZ FILHO, (1996) e MALHADO et al. (2001). Através da análise estatística pode-se constar que as regiões diferem entre si, como podemos observar na FIGURA 8, sendo significativo para todos os pesos.

O efeito da região sobre o peso aos 205 dias foi significativo $(\mathrm{P}<0,001)$, conforme FIGURA 8, a variação observada é favorável à região Bodoquena, em segundo lugar à região Iguatemi, ficando a região de Dourados, com os menores.

FIGURA 8 - MÉDIA DOS PESOS AOS 205(P205), 365 (P365) E 550 (P550) DIAS DE IDADE, DE ACORDO COM AS REGIÕES DE CRIAÇÃO EM BOVINOS DA RAÇA NELORE MOCHA, SUDOESTE DE MATO GROSSO DO SUL (1975-2001).

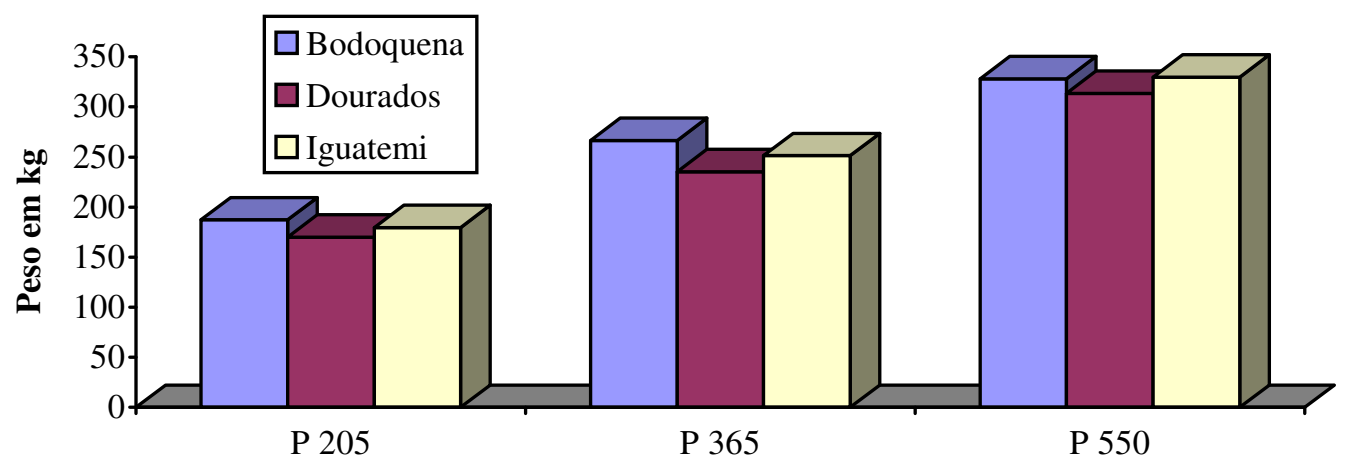


Fatores ambientais que influenciam o peso à desmama, ano e sobreano em bovinos da raça nelore mocha...

Considerando o peso aos 365 dias de idade, à região Bodoquena foi a que apresentou a maior média, em segundo lugar, foi a região lguatemi, e em último a região Dourados, conforme observado na FIGURA 8. O efeito da região sobre o peso aos 550 teve como resultados a Região 3 com a maior média de peso, em segundo a Região, e em último a Região 2, conforme observado na FIGURA 8.

Com base nos resultados obtidos neste estudo, verifica-se que a melhor região de produção de animais Nelore Mocha, é a região Bodoquena, exceto para o peso aos 550 dias em que a Região Iguatemi foi melhor. As diferenças entre condições edafoclimáticas, espécies forrageiras, manejos e constituições genéticas, em conjunto ou isoladamente, são fatores que podem ter contribuído para estas diferenças entre as regiões. Tais diferenças também foram verificadas por TAYLOR (1960) nos Estados Unidos, STRAW e JONES (1977) na Austrália, SILVA (1990) no Brasil appud FERRAZ FILHO, 1996 e SOUZA, 1997. Os efeitos significativos da região de criação sobre os pesos, indicam a necessidade de um ajuste prévio, por ocasião da comparação de animais nascidos em diferentes regiões, da avaliação de reprodutores e da obtenção de parâmetros genéticos, na raça Nelore Mocho (FERRAZ FILHO, 1996).

Quanto a idade da vaca ao parto, estas apresentaram melhor produtividade com média de 8,8 anos para o peso aos 205 dias de idade, e 8,5 anos paro o peso aos 365 dias de idade, influenciando $(P<0,001)$ os pesos na forma linear e quadrática. Porem, para o peso aos 550 dias de idade não houve efeito significativo $(P>0,05)$. Esses resultados indicam que a influência da idade da vaca sobre o peso dos bezerros fez sentir mesmo após a desmama, refletindo a capacidade materna. A idade da vaca de máxima produção, foi cerca de 106; 102,58 e 96,49 meses, considerando os pesos aos 205, 365 e 550 dias de idade, respectivamente.

Nas FIGURAS 9 e 10 são apresentadas às curvas de regressão dos pesos aos 205 e 365 dias de idade, em função da idade da vaca ao parto, assim como as respectivas equações de previsão. Os valores obtidos mostram que os pesos aumentaram à medida que a idade da vaca cresceu, até atingir um máximo, e depois os pesos decresceram. A idade da vaca para o peso aos 550 dias de idade não foi significativa.

\section{FIGURA 9 - EFEITO DA IDADE DA VACA SOBRE O PESO AOS 205 (P205) DIAS DE IDADE EM BOVINOS DA RAÇA NELORE MOCHO, SUDOESTE DE MATO GROSSO DO SUL (1975-2001).}

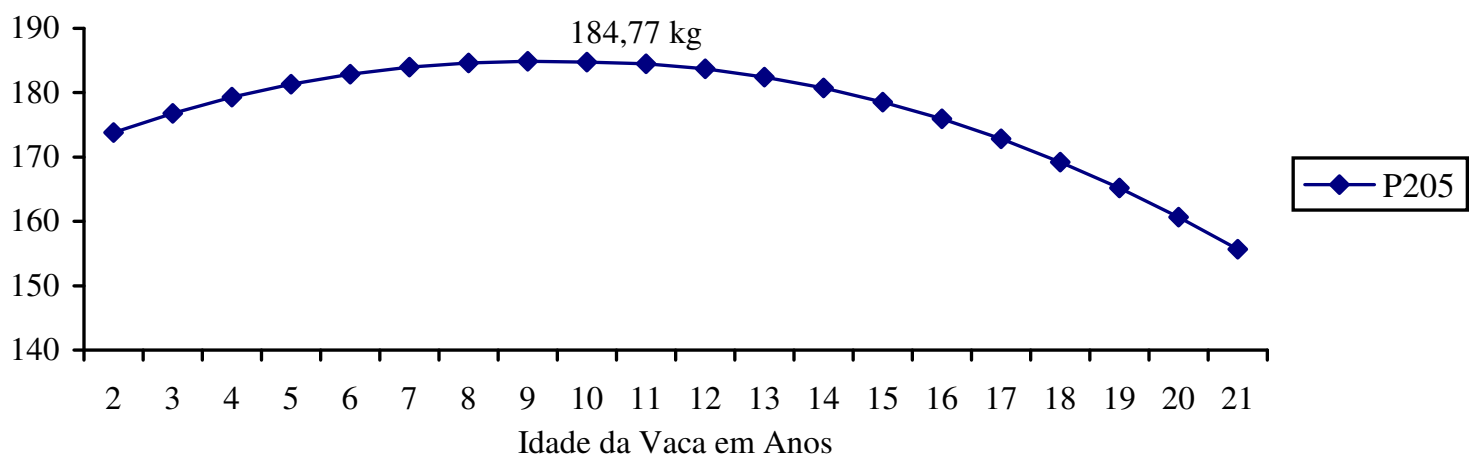

Através dos dados obtidos, podemos observar que a influência da idade da mãe indica que variações no ambiente pré e pós-natal, decorrem da variação da idade da vaca, influindo nos pesos aos 205 e 365 dias de idade, refletindo ainda nos pesos aos 550 dias de idade, uma vez que as primíparas geralmente estão, ainda, em fase de desenvolvimento e tem um peso corporal inferior ao que atingirão em sua maturidade, e, portanto, tendem a parir bezerros mais leves. Contudo em uma idade mais avançada, tendem a parir bezerros, mais leves. Esta influencia também foi observada com efeito significativo por WAUGH e MARLOEW (1969), SILVA (1990), FERRAZ FILHO (1996) e SOUZA (1997). Por outro lado ROSA (1977) e TORRES et al., (1979), não apontaram influência da vaca na fase de recria ou mesmo na de cria. 
FIGURA 10 - EFEITO DA IDADE DA VACA SOBRE O PESO AOS 365 (P365) DIAS DE IDADE EM BOVINOS DA RAÇA NELORE MOCHO, SUDOESTE DE MATO GROSSO DO SUL (1975-2001).

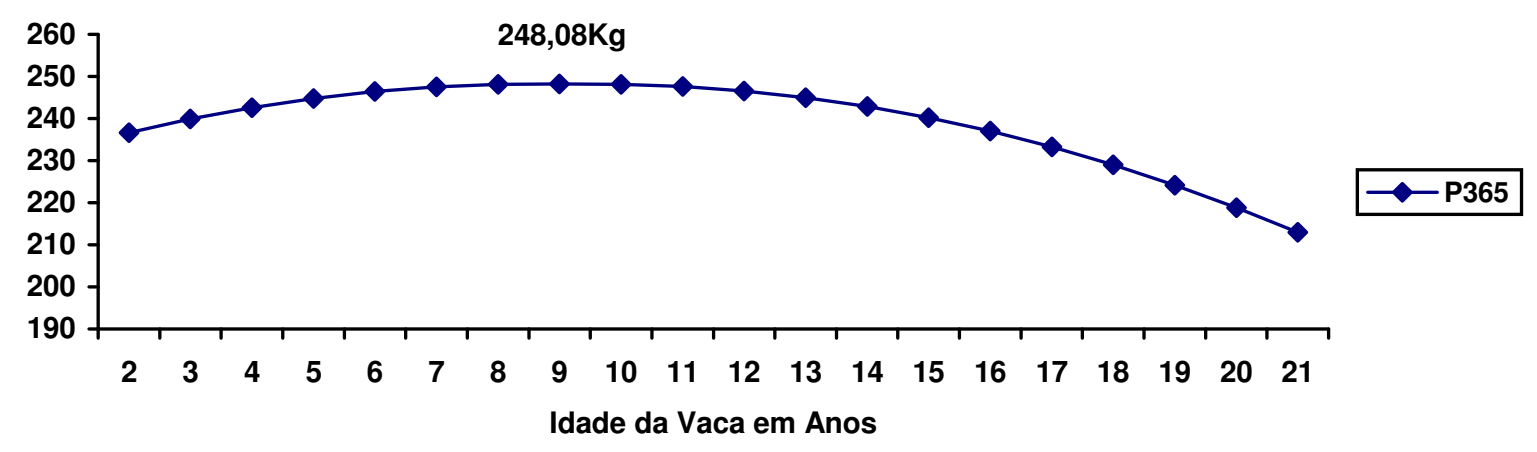

\section{Conclusão}

Os resultados significativos de efeito de meio (região, mês e ano de nascimento, sexo e idade da vaca ao parto), encontrados sobre os pesos aos 205,365 e 550 dias de idade indicam a necessidade de ajuste prévio visando suprir a influência do meio. Estes efeitos são importantes na determinação de características produtivas, portanto, servirão como subsídios para estudos de avaliações genéticas em bovinos da raça Nelore Mocho.

\section{REFERÊNCIAS}

ELER, J.P.; LÔBO, R.B.; ROSA, A.N. Influência de fatores genéticos e de meio em pesos de bovinos da raça Nelore criados no Estado de São Paulo. Revista da Sociedade Brasileira de Zootecnia, Viçosa, v. 18, n. 2, p. 103-1111, 1989.

EUCLIDES FILHO, K.; NOBRE, P.R.C.; ROSA, A.N. Idade da vaca e suas interrelações com a fazenda, reprodutor e sexo do bezerro. Revista da Sociedade Brasileira de Zootecnia, Viçosa, v. 20, p. 40-6, 1991.

FERRAZ FILHO, P.B. Análise e tendência genética de pesos em bovinos da raça Nelore mocha no Brasil. Jaboticabal, 1996. 163 f. Dissertação (Mestrado) - Universidade Estadual Paulista.

FERRAZ FILHO, P.B. Avaliação genética do desenvolvimento ponderal de bovinos da raça Tabapuã no Brasil. Botucatu, 2001. 135 f. Tese (Doutorado em Genética) - Universidade Estadual Paulista.

FERRAZ FILHO, P.B.; RAMOS, A.A.; SILVA, L.O.C.; SOUZA, J.C; ALENCAR, M.M. Environmental and genetic influencing pré and post-weaning growth traits of tabapuã cattle in Brazil. Archives of Veterinary Science, Curitiba, v. 6, n. 2, p. 19-30, 2001.
GRESSLER, S. L. Estudo de fatores ambientais e parâmetros genéticos de algumas características reprodutivas em animais da raça Nelore. Belo Horizonte, 1998. 150 p. Dissertação (Mestrado) - Escola de Veterinária, Universidade Federal de Minas Gerais.

INSTITUTO BRASILEIRO DE GEOGRAFIA E ESTATÍSTICA. Anuário Estatístico do Brasil. 2003. Disponível em: <www.ibge.gov.br>. Acesso em:jun. 2005.

MALHADO, C.H.M.; SOUZA, J.C.; SILVA, L.O.C. et al. Influência da época de nascimento sobre as percentagens de crescimento do nascimento aos 550 dias de idade em bovinos da raça Guzerá criados em duas regiões brasileira. In: REUNION LATINO AMERICANA DE PRODUCION ANIMAL, 17., 2001, Cuidad de la Habana. No prelo.

MARTINS, G.A.; MARTINS FILHO, R.; LOBÔ, R.N.B. Fatores genéticos e de ambiente que influenciam o peso à desmama em bovinos da raça Nelore. In: REUNIÃO NANUAL DA SOCIEDADE BRASILEIRA DE ZOOTECNIA, 33, 1996, Fortaleza. Anais... Fortaleza: SBZ, 1996. p. 181-183.

MASCIOLI, A.S.; PAS, C.C.P.; EL FARO, L. et al. Estimativas de parâmetros genéticos e fenotípicos para características de crescimento até a desmama em bovinos da raça Canchin. Revista da Sociedade Brasileira de Zootecnia, Viçosa, v. 26, n. 4, p. 709-713, 1997.

MILAGRES, J.C.; ARAUJO, C.R.; TEIXEIRA, N.M.; TORRES, R.A. Influencias de meio e de herança sobre os pesos ao nascer, aos 205 e aos 365 dias de idade de animais Nelore criados no nordeste do Brasil. Revista da Sociedade Brasileira de Zootecnia, Viçosa, v. 22, n. 3, p. 455-465, 1993.

NOBRE, P.R.C.; ROSA, A.N.; SILVA, L.O.C. Influência de fatores genéticos e de meio sobre os pesos de gado Nelore no estado da Bahia - Brasil. Revista da Sociedade Brasileira de Zootecnia, Viçosa, v. 14, p. 338-57, 1985. 
Fatores ambientais que influenciam o peso à desmama, ano e sobreano em bovinos da raça nelore mocha...

ROSA, A.N. Análise genética de parâmetros de crescimento e proposição de índices de seleção para animais da raça Nelore. Viçosa, 1977. 67 p. Dissertação (Mestrado) - Universidade Federal de Viçosa.

ROSA, A.N.; SILVA, L.O.C.; NOBRE, P.R.C. Avaliação do desempenho de animais Nelore em controle do desenvolvimento ponderal no Estado do Mato Grosso do Sul- Brasil. Revista da Sociedade Brasileira de Zootecnia, Viçosa, v. 15, n. 6, p. 515-32, 1986.

SAS. Statistical Analysis System - User Guide: Stat, Version 6.11. Cary, (NC: Sas Institute Inc.) 2001.

SILVA L.O.C.; ROSA, A.N.; NOBRE, P.R.C.; MILAGRES, J.C.; EVANGELISTA,S.R.M. Análise de pesos de bovinos Nelore criados a pasto no Estado de São Paulo, Brasil. Pesquisa Agropecuária Brasileira, Brasília, v. 22, n. 11/12, p. 1245-1256, 1987.

SILVA, L.O.C. Tendência genética e interação genótipo $x$ ambiente em rebanhos Nelore, criados a pastos no Brasil Central. Viçosa, 1990. $113 \mathrm{f}$. Tese (Doutorado)- Universidade Federal de Viçosa.

SOUZA, J.; BRÜLÉ, A.O.; FERRAZ FILHO, P.B.; OLIVIERA, J.A.; ALENCAR, M.M. Repetibilidade dos pesos e ganho de peso, do nascimento à desmama, de bovinos da raça Nelore. Revista da Sociedade Brasileira de Zootecnia, Viçosa, v. 23, n. 1, p. 133-139, 1994.

SOUZA, J.C.; RAMOS, A.A. Efeitos de fatores genéticos e do meio sobre os pesos de bovinos da raça Nelore. Revista da Sociedade Brasileira de Zootecnia, Viçosa, v. 24, n. 1, p. 167-172, 1995.
SOUZA, J.C. Interação genótipo $x$ ambiente sobre o peso ao desmame de zebuínos da raça Nelore no Brasil. Botucatu, 1997. 121 p. Tese (Doutorado em Genética) - Universidade Estadual Paulista.

SOUZA, J.C., SILVA, L.O.C., FERRAZ FILHO, P.B. et al. Estimativas de parâmetros e da tendência genética para o peso aos 205 dias de idade de zebuínos da raça Guzerá. In: REUNIÃO ANUAL DA SOCIEDADE BRASILEIRA DE ZOOTECNIA, 37, 2000, Viçosa. Anais... Viçosa: SBZ, 2000. p. 220.

STRAW, W.M.; JONES, L.P. Estimation of environmental effects on post-weaning aging and yearling weight of beef cattle. Australian Journal of Experimental Agriculture and Animal Husbandry, Melbourne, v. 17, n. 89, p. 885-891, 1977.

TAYLOR, J.C.; CARTER, R.C. KINCAID, C.M.; PRIODE, B.M; GAINES, J.A. Estimates of genetic an phenotypic parameters in beef cattle. Journal of Animal Science, Savoy, v. 19, n. 3, p. 101$108,1960$.

TORRES, R.A.A.; SILVA, M.A.; TORRES, J.R. Fatores de meio e herança que afetam os pesos e o ganho de peso de bezerros Gir na fase de aleitamento. Revista da Sociedade Brasileira de Zootecnia, Viçosa, v. 8, n. 3, p. 488-496, 1979.

WAUGH, G.A.; MARLOEW, T.J. Environmental influences on growth rate and grade of yearling beef cattle. Journal of Animal Science, Savoy, v. 29, n. 4, p. 541-546, 1969.

Recebido para publicação: 30/06/2005

Aprovado: $26 / 10 / 2005$ 